\title{
COMMUTATIVE ALGEBRAS SATISFYING AN IDENTITY OF DEGREE FOUR
}

\author{
J. MARSHALL OSBORN ${ }^{1}$
}

In another paper [2] we have established the following result:

Theorem. Let $A$ be a commutative (nonassociative) algebra with unity element over a field of characteristic not 2 or 3 , and let A satisfy an identity of degree $\leqq 4$ not implied by the commutative law. Then $A$ satisfies at least one of the following three identities:

$$
\begin{array}{cc}
\left(x^{2} x\right) x & =x^{2} x^{2}, \\
\text { (2) } & 2(y x \cdot x) x+y x^{8}=3\left(y x^{2}\right) x \\
\text { (3) } & 2\left(y^{2} x\right) x-2(y x \cdot y) x-2(y x \cdot x) y+2\left(x^{2} y\right) y-y^{2} x^{2}+(y x)(y x)=0 .
\end{array}
$$

In view of this theorem, the study of the structure of commutative algebras with unity element satisfying an identity of degree $\leqq 4$ is immediately reduced to the study of algebras satisfying one of the identities (1)-(3). The first of these identities is well-known to be equivalent to power-associativity in a commutative algebra of characteristic not 2,3 , or 5 and has been studied extensively [1]. The identities (2) and (3) do not seem to have been investigated in the literature.

The purpose of the present paper is to study commutative rings which satisfy (2). As we shall see in $\S 1$, this identity also arises in a very natural way as a consequence of the Jordan identity, $\left(x^{2} y\right) x$ $=x^{2}(y x)$. From this it is not difficult to see that a commutative ring of characteristic relatively prime to 2 or 3 satisfies the Jordan identity if and only if it satisfies both (1) and (2). For any characteristic one can find commutative algebras with unity element satisfying either (1) or (2), but which do not satisfy the Jordan identity. Thus the class of commutative rings satisfying (2) is strictly larger than the class of Jordan rings and is not included in the class of power-associative rings. The main results of this paper are summarized by the following two theorems:

THEOREM 1. Let A be a commutative ring satisfying (2) of characteristic relatively prime to 2 , and let $A$ contain an idempotent $e$ which is not a unity element. Then $A$ is a Jordan ring if and only if $A_{e}(1)$ and $A_{e}(0)$

Received by the editors August 1, 1963 and, in revised form, July 23, 1964.

1 The research for this paper was supported by National Science Foundation Grant G-19052. 
are Jordan rings. In particular, if $A$ is simple, then it is a Jordan ring.

Theorem 2. Let $A$ be a finite-dimensional algebra satisfying (2) of characteristic not 2, and let every nonzero ideal of $A$ contain an idempotent. Then $A$ has a unity element and is the direct sum of simple algebras.

In view of these results, the study of commutative algebras satisfying (2) is largely reduced to the question of determining the algebras of degree one satisfying (2). It would also be interesting to know whether a finite-dimensional algebra $A$ satisfying (2) necessarily contains a unique ideal $N$ which is maximal with respect to the property of not containing an idempotent, and whether $A_{e}(1 / 2)+A_{e}(0)$ is contained in $N$ for any principal idempotent $e$ of $A$.

1. Preliminary results. Linearizing (1) completely, we obtain

$$
2 \sum^{6}(y x \cdot z) w+2 \sum^{3} y(x z \cdot w)=6 \sum^{3}(y \cdot x z) w
$$

where each summation stands for the sum of all distinct terms obtained from the given term by permuting $x, z$ and $w$. Setting $H(y ; x, z, w)=(y \cdot x z) w+(y \cdot z w) x+(y \cdot w x) z$, we may write $(4)$ in the form

$$
H(z ; x, y, w)+H(w ; x, y, z)+H(x ; y, z, w)=3 H(y ; x, z, w)
$$

after dividing by 2 . Adding $H(y ; x, z, w)$ to this equation, we get

$$
\begin{gathered}
H(y ; x, z, w)+H(z ; x, y, w)+H(w ; x, y, z)+H(w ; x, y, z) \\
=4 H(y ; x, z, w)
\end{gathered}
$$

the left side of which is symmetric in $x, y, z, w$. Thus, for characteristic relatively prime to 2 , the function $H$ is symmetric in its arguments, giving

$$
H(x ; y, z, w)=H(y ; x, z, w)
$$

or

(5) $(x \cdot y z) w+(x \cdot z w) y+(x \cdot w y) z=(y \cdot x z) w+(y \cdot z w) x+(y \cdot w x) z$.

Setting $w=z=x$ in (5) yields (2) again, so that (5) is equivalent to (2) for characteristic relatively prime to 2 .

Since (5) is an immediate consequence of the linearized Jordan identity, the class of rings studied here includes the class of Jordan rings. On the other hand using (5), the linearized power-associative 
identity may be reduced to the Jordan identity, so that our class of rings doesn't include any power-associative rings which are not already Jordan rings. The class of rings satisfying (2) are not all Jordan rings, however, since it was shown in [2] that there exists a commutative algebra with unity element over any field of characteristic not 2 or 3 satisfying (2) but not $x^{3} x=x^{2} x^{2}$.

Next, letting $x=e$ (an idempotent) in (2), we get the relation $2 R_{e}^{3}-3 R_{e}^{2}+R_{e}=0$, or $R_{e}\left(2 R_{e}-I\right)\left(R_{e}-I\right)=0$. Defining $A_{\lambda}=A_{e}(\lambda)$ $=\{x \mid x \in A, x e=\lambda x\}$, it is easy to check that each $A_{e}(\lambda)$ is an additive subgroup of $A$, and that we have the additive direct sum decomposition

$$
A=A_{\odot}(1)+A_{\odot}(1 / 2)+A_{\odot}(0)
$$

for each idempotent $e$ of $A$. To discover what can be said about products of the $A_{\lambda}$ 's, we set $x=w=e, y e=j y, z e=k z$ in (5) to get $(y z) R_{c}^{2}$ $+k^{2}(y z)+j^{2}(y z)=2 k(y z) R_{e}+j(y z)$, or

$$
(y z)\left[R_{\bullet}^{2}-2 k R_{\bullet}+\left(k^{2}+j^{2}-j\right) I\right]=0 .
$$

Setting $j$ and $k$ equal to $0,1 / 2,1$ in all possible ways in (7), we get a set of relations which can be restated as

LEMMA 1. For a fixed e, products between the $A_{\lambda}$ 's are governed by the relations $A_{1}^{2} \subset A_{1}, A_{0}^{2} \subset A_{0}, A_{1} A_{0}=0, A_{1} A_{1 / 2} \subset A_{1 / 2}, A_{0} A_{1 / 2} \subset A_{1 / 2}$, and $A_{1 / 2}^{2} \subset A_{1}+A_{0}$.

If $u$ and $v$ are orthogonal idempotents, we see from this lemma that $A_{v}(1) \subset A_{u}(0), A_{u}(1 / 2) R_{v} \subset A_{u}(1 / 2)$, and $A_{u}(0) R_{v} \subset A_{u}(0)$. Hence for $y \in A_{u}(1 / 2)$, we get $y v \cdot u=\frac{1}{2} y v=y u \cdot v$. Similarly $y v \cdot u=y u \cdot v$ holds for $y$ in $A_{u}(1)$ or $A_{u}(0)$, to give the relation $R_{v} R_{u}=R_{u} R_{v}$ in the ring $A$. This leads easily to the usual simultaneous decomposition of $A$ with respect to two or more orthogonal idempotents which is familiar from Jordan theory.

To find out more information about how elements of the $A_{\lambda}$ 's multiply, we now set $w=e, x e=i x, y e=j y, z e=k z$ in (5) to obtain

$$
(x \cdot y z) R_{e}+k(x z \cdot y)+j(x y \cdot z)=(y \cdot x z) R_{e}+k(y z \cdot x)+i(y x \cdot z),
$$

or

$$
(y, z, x)\left[R_{\bullet}-k I\right]+(j-i)(x y \cdot z)=0 .
$$

Selecting appropriate values for $i, j, k$ and adding subscripts to our symbols to indicate which $A_{\lambda}$ they are assumed to belong to, equation (8) yields the relations given in 
Lemma 2. Products of elements from different $A_{\lambda}$ 's satisfy the following relations:

$$
\begin{aligned}
y_{1 / 2}\left(x_{1} z_{1}\right) & =\left(y_{1 / 2} x_{1}\right) z_{1}+\left(y_{1 / 2} z_{1}\right) x_{1}, \\
y_{1 / 2}\left(x_{0} z_{0}\right) & =\left(y_{1 / 2} x_{0}\right) z_{0}+\left(y_{1 / 2} z_{0}\right) x_{0} \\
x_{1}\left(y_{1 / 2} z_{1 / 2}\right) & =\left[\left(x_{1} y_{1 / 2}\right) z_{1 / 2}+\left(x_{1} z_{1 / 2}\right) y_{1 / 2}\right]_{1}, \\
x_{0}\left(y_{1 / 2} z_{1 / 2}\right) & =\left[\left(x_{0} y_{1 / 2}\right) z_{1 / 2}+\left(x_{0} z_{1 / 2}\right) y_{1 / 2}\right]_{0}, \\
{\left[\left(x_{1} y_{1 / 2}\right) z_{1 / 2}\right]_{0} } & =\left[\left(x_{1} z_{1 / 2}\right) y_{1 / 2}\right]_{0}, \\
{\left[\left(x_{0} y_{1 / 2}\right) z_{1 / 2}\right]_{1} } & =\left[\left(x_{0} z_{1 / 2}\right) y_{1 / 2}\right]_{1}, \\
\left(x_{1} y_{1 / 2}\right) z_{0} & =x_{1}\left(y_{1 / 2} z_{0}\right) .
\end{aligned}
$$

2. Proof of Theorem 1. If we define $K(x, y, z, w)=(x y)(z w)$ $+(x z)(y w)+(x w)(y z)$, the ring $A$ will be Jordan if and only if

$$
H(x ; y, z, w)=K(x, y, z, w)
$$

for all ways of choosing $x, y, z, w$ in $A_{1}, A_{1 / 2}, A_{0}$. We begin with the case $x \in A_{1 / 2}$ and $y, z, w \in A_{1}$. Here, $K(x, y, z, w)=(y x)(z w)+(x z)(y w)$ $+(x w)(y z)=(x y \cdot z) w+(x y \cdot w) z+(x z \cdot y) w+(x z \cdot w) y+(x w \cdot y) z$ $+(x w \cdot z) y=(x \cdot y z) w+(x \cdot y w) z+(x \cdot w z) y=H(x ; y, z, w)$, all using just the first equation of (9).

Suppose next that $x, y \in A_{1 / 2}$ and $z, w \in A_{1}$. Subtracting and adding two terms and splitting two others into components, we get

$$
\begin{aligned}
& (x y)(z w)+(x z)(y w)+(x w)(y z) \\
& (14)=\left\{(x y)(z w)-[(y z \cdot w) x]_{1}-[(y w \cdot z) x]_{1}+[(x w)(y z)+(x z)(y w)]_{0}\right\} \\
& +\left\{[(x w)(y z)]_{1}+[(y z \cdot w) x]_{1}\right\}+\left\{[(x z)(y w)]_{1}+[(y w \cdot z) x]_{1}\right\} .
\end{aligned}
$$

Since $y z \cdot w+y w \cdot z=y \cdot z w$ and $[(x w)(y z)+(x z)(y w)]_{0}=[(y z \cdot w) x$ $+(y w \cdot z) x]_{0}=[(y \cdot z w) x]_{0}$ using (9) and (11), the expression within the first braces in (14) reduces to $(y x)(z w)-[(y \cdot z w) x]_{1}+[(y \cdot z w) x]_{0}$ $=[(x \cdot z w) y]_{1}+[(x \cdot z w) y]_{0}=(x \cdot z w) y$. On the other hand, the expressions within the second and third set of braces of (14) are $(x \cdot y z) w$ and $(x \cdot y w) z$ respectively using $(10)$.

For the case $x \in A_{1}$ and $y, z, w \in A_{1 / 2}$, we must first set $x=e$ and $y, z, w \in A_{1 / 2}$ in (5) to obtain

$$
(y z) R_{\bullet} \cdot w+(z w) R_{\bullet} \cdot y+(y w) R_{\bullet} \cdot z=\frac{1}{2}\{(y z) w+(z w) y+(y w) z\},
$$

or

(15) $(y z)_{1} \cdot w+(z w)_{1} \cdot y+(y w)_{1} \cdot z=(y z)_{0} \cdot w+(z w)_{0} \cdot y+(y w)_{0} \cdot z$.

Using (9), (12) and (15), we can now establish (13): 


$$
\begin{aligned}
(x w)(y z)_{1}+(x w)(y z)_{0} & +(x y)(z w)_{1}+(x y)(z w)_{0}+(x z)(y w)_{1}+(x z)(y w)_{0} \\
= & (x w)(y z)_{1}+\left[(y z)_{0} \cdot w\right] x+(x y)(z w)_{1}+\left[(z w)_{0} \cdot y\right] x \\
& +(x z)(y w)_{1}+\left[(y w)_{0} \cdot z\right] x \\
= & (x w)(y z)_{1}+\left[(y z)_{1} \cdot w\right] x+(x y)(z w)_{1}+\left[(z w)_{1} \cdot y\right] x \\
& +(x z)(y w)_{1}+\left[(y w)_{1} \cdot z\right] x \\
= & (x \cdot y z) w+(x \cdot z w) y+(x \cdot y w) z .
\end{aligned}
$$

Let us consider next the case when all four variables are in $A_{1 / 2}$. By symmetry, we need only check the component in $A_{1}$ :

$$
\begin{aligned}
&(x y)(z w)_{1}+(x z)(y w)_{1}+(x w)(y z)_{1} \\
&= {\left[\left((z w)_{1} \cdot x\right) y+\left((z w)_{1} \cdot y\right) x\right]_{1}+\left[\left((y w)_{1} \cdot x\right) z+\left((y w)_{1} \cdot z\right) x\right]_{1} } \\
&+\left[\left((y z)_{1} \cdot x\right) w+\left((y z)_{1} \cdot w\right) x\right]_{1} \\
&= {\left[\left((z w)_{1} \cdot x\right) y+\left((z w)_{0} \cdot y\right) x\right]_{1}+\left[\left((y w)_{1} \cdot x\right) z+\left((y w)_{0} \cdot z\right) x\right]_{1} } \\
&+\left[\left((y z)_{1} \cdot x\right) w+\left((y z)_{0} \cdot w\right) x\right]_{1} \\
&= {\left[\left((z w)_{1} \cdot x\right) y+\left((z w)_{0} \cdot x\right) y\right]_{1}+\left[\left((y w)_{1} \cdot x\right) z+\left((y w)_{0} \cdot x\right) z\right]_{1} } \\
&+\left[\left((y z)_{1} \cdot x\right) w+\left((y z)_{0} \cdot x\right) w\right]_{1} \\
&= {[(x \cdot z w) y]_{1}+\left[(x \cdot y w)_{1}\right]_{1}+[(x \cdot y z) w]_{1} . }
\end{aligned}
$$

We have now established (13) for all cases in which the arguments come from $A_{1}$ and $A_{1 / 2}$, except for the case when all four arguments lie in $A_{1}$. By symmetry, (13) must also hold when the arguments come from $A_{0}$ and $A_{1 / 2}$, except when they all lie in $A_{0}$. To prove the first assertion of Theorem 1 , it remains to show (13) in those cases which involve $A_{1}, A_{1 / 2}$, and $A_{0}$ all at once. But if $x \in A_{1 / 2}, w \in A_{0}$, and $y, z \in A_{1}$, then $K(x, y, z, w)=(x w)(y z), H(x ; y, z, w)=(x \cdot y z) w$, and the two are equal by (12). The case with one argument each in $A_{1 / 2}$ and $A_{1}$ and two arguments in $A_{0}$ follows by symmetry. And finally, if $x, y \in A_{1 / 2}, z \in A_{1}$ and $w \in A_{0}$ we compute

$$
\begin{aligned}
(x y)(z w)+(x z) & (y w)+(x w)(y z) \\
& =[(x z)(y w)]_{0}+[(x z)(y w)]_{1}+[(x w)(y z)]_{0}+[(x w)(y z)]_{1} \\
& =[(w y \cdot z) x]_{0}+[(x w)(y z)]_{0}+[(x z)(y w)]_{1}+[(z y \cdot w) x]_{1} \\
& =[(w \cdot y z) x]_{0}+[(x w)(y z)]_{0}+[(x z)(y w)]_{1}+[(z \cdot y w) x]_{1} \\
& =[(x \cdot y z) w+(x \cdot y w) z+(x \cdot z w) y .
\end{aligned}
$$

To prove the last part of Theorem 1, it suffices to show that $A_{1}$ and $A_{0}$ are Jordan rings if $A$ is simple. But (9) induces a homomorphism of $A_{1}$ into the ring of all Jordan endomorphisms on $A_{1 / 2}$, and the 
kernel $C_{1}$ of this homomorphism is an ideal of $A_{1}$ which annihilates both $A_{1 / 2}$ and $A_{0}$. Hence $C_{1}$ is an ideal of $A$. Since $e$ is not the unity element of $A$ by hypothesis, we have $C_{1} \subset A_{1} \neq A$, showing that $C_{1}=0$ and that $A_{1}$ is a Jordan ring. By symmetry, the simplicity of $A$ also implies that $A_{0}$ is a Jordan ring.

Suppose now that $e$ is the sum of the orthogonal idempotents $e^{\prime}$ and $e^{\prime \prime}$. Then Theorem 1 implies that $A_{e}(1)$ is a Jordan ring if and only if $A_{e^{\prime}}(1)$ and $A_{e^{\prime \prime}}(0)$ are Jordan rings, and hence $A$ is a Jordan ring if and only if $A_{e^{\prime}}(1), A_{e^{\prime \prime}}(1)$, and $A_{e}(0)$ are Jordan rings. By inducting on this argument it is easy to establish the following

CoROllary. Let $A$ be a commutative ring satisfying (2) of characteristic relatively prime to 2 , and let $A$ contain the mutually orthogonal idempotents $e_{1}, \cdots, e_{n}$ for $n \geqq 2$. Then $A$ is a Jordan ring if and only if $A_{e_{1}}(1), \cdots, A_{e_{n}}(1)$, and $\bigcap_{i=1}^{n} A_{e_{i}}(0)$ are all Jordan rings.

3. Proof of Theorem 2. Let $A$ be a finite-dimensional algebra satisfying (2) of characteristic not 2 , and suppose that some minimal ideal $B$ of $A$ contain an idempotent. If $e_{1}, \cdots, e_{n}$ are a maximal set of mutually orthogonal idempotents in $B$, then $e=e_{1}+\cdots+e_{n}$ is a principal idempotent of $B$. Letting $B=B_{1}+B_{1 / 2}+B_{0}$ be the decomposition of $B$ with respect to $e$, we observe that the decomposition of $A$ with respect to $e$ is given by $A=B_{1}+B_{1 / 2}+A_{0}$, since $e \in B$. As in the proof of Theorem 1 , we see that $B_{1}$ and $A_{0}$ may be represented as Jordan endomorphisms on $B_{1 / 2}$ using (9) and that the kernels $C_{1}$ and $C_{0}$ of these representations are ideals of $A$. But the ideals $C_{1}$ and $C_{0} \cap B$ of $A$ are contained in $B$, so that $C_{0} \cap B=0$ and either $C_{1}=B$ or $C_{1}=0$.

Suppose first that $C_{1}=0$. Then $B_{1}$ and $B_{0}$ are Jordan algebras, and hence $B$ is a Jordan algebra by Theorem 1 . Using the standard theory of Jordan algebras, we may conclude that $B$ contains a unique ideal $M$ maximal with respect to the property of not containing any idempotents, and that $B_{1 / 2}+B_{0} \subset M$. But then $M A \subset M B_{1}+M B_{1 / 2}+M A_{0}$ $\subset M+B_{1 / 2} A_{0}+B_{0} A_{0} \subset M+B_{1 / 2}+B_{0} \subset M$, showing that $M$ is an ideal of $A$. Since $B$ is a minimal ideal of $A$, we have $M=0$ and $B_{1 / 2}+B_{0}=0$. Observing that the case $C_{1}=B$ also leads to $B_{1 / 2}+B_{0}=0$, we see that, in either case, $e$ is the unity element of $B$ and $A=B \oplus A_{0}$. We have proved that any minimal ideal containing an idempotent in a finitedimensional algebra satisfying (2) is a direct summand.

It is now easy to establish Theorem 2 by induction on the dimension of $A$. If every ideal of $A$ contains an idempotent, so does every ideal of $A_{0}$, and hence $A_{0}$ has a unity element $f$ and is a direct sum of simple algebras by inductive hypothesis. Then $e+f$ is a unity ele- 
ment for $A$, and since $B$ is simple, $A$ is also a direct sum of simple algebras.

\title{
REFERENCES
}

1. A. A. Albert, $A$ theory of power-associative commutative algebras, Trans. Amer. Math. Soc. 69 (1950), 503-527.

2. J. M. Osborn, Identities on nonassociative algebras, Canad. J. Math. 17 (1965), 78-92.

UNIVERSITY OF WISCONSIN

\section{SOME PROPERTIES OF LOCALIZATION AND NORMALIZATION}

\author{
JOSEPH LIPMAN
}

In a recent note [1], S. Abhyankar has given some lemmas concerning localization and normalization for noetherian rings without nilpotent elements. We give a characterization of those rings in which every prime ideal is maximal (Proposition 1) and deduce generalizations of Abhyankar's results (cf. Corollary 1 and Corollary 2).

Preliminaries. A ring will always be a nonnull commutative ring with identity.

For properties of rings of quotients see [3, $\$ \$ 9-11$ of Chapter IV]. Recall that if $R$ is a ring with total quotient ring $K$, and if $M$ is a multiplicative system in $R$, then we may identify the ring of quotients $R_{M}$ with a subring of $K_{M}$. When this is done, the total quotient ring of $K_{M}$ is also the total quotient ring of $R_{M}$.

Denote by $g_{M}$ the canonical map of $K$ into $K_{M}$; the restriction of this map to $R$ is then the canonical map of $R$ into $R_{M}$; the restricted map may also be denoted by $g_{M}$ without fear of confusion. If $M$ consists of all the powers of a single element $f$, then we write $R_{f}, K_{f}, g_{f}$, in place of $R_{M}, K_{M}, g_{M}$.

If $Q$ is a minimal prime ideal in $R$, and $M$ is the complement of $Q$ in $R$, then $Q R_{M}$, being the only prime ideal in $R_{M}$, consists entirely of zerodivisors (in fact, of nilpotents). Consequently, if $x \in Q$, then $g_{M}(x)$ is a zerodivisor, and it follows easily that $x$ is a zerodivisor. Thus any minimal prime ideal in a ring consists entirely of zerodivisors.

Proposition 1. For a ring $R$, the following statements are equivalent: (1) Every prime ideal in $R$ is maximal.

Received by the editors July 2, 1964. 